\title{
T-regulatory/T-helper 17: promiscuous signals and fear of commitment
}

Evaluation of: Yang XO, Nurieva R, Martinez GJ et al.: Molecular antagonism and plasticity of regulatory and inflammatory T cell programs. Immunity 29, 44-57 (2008). The insight gained recently on the differentiation of naive CD4 T cells challenges the dogma that expression of canonical transcription factors and production of signature cytokines portray a commitment to a specific lineage and a point of no return. For almost two decades now, the belief has been that naive CD4 T cells, under the guidance of environmental signals, follow a one-way road to evolve as Th1, Th2, Th17 or regulatory T cells (Tregs). The current paper, however, demonstrates a crosstalk between signals and identifies transitory T-cell states whereby a differentiating $\mathrm{CD}^{+}{ }^{+} \mathrm{T}$ cell will express a mixed Th17 and Treg phenotype. Moreover, they were able to successfully reprogram terminally differentiated Tregs into Th17 cells, suggesting that redifferentiation could occur and provides an environmental plasticity to readjust immunity. This suggests that T-cell differentiation does not necessarily follow a one-way road, but that traffic may flow in both directions.

KEYWORDS: T-helper 17 - T-helper cell differentiation - T-regulatory cell

Regulatory T cells (Tregs) were identified as suppressive lymphocytes capable of impeding immune responses [1,2]. Expression of the FoxP3 transcription factor is thought to be critical for the development of Tregs [3], and many different surface molecules have been implicated in their function [4]. Expression of FoxP3 was regarded as a definite commitment to the Treg lineage and a prior report demonstrated a struggle between FoxP 3 and the AP-1 transcription factor to form a FoxP3:NFAT instead of an AP1:NFAT complex to direct naive $T$ cells towards Tregs [5] and away from an activated effector phenotype. TGF- $\beta$ was the first environmental cytokine defined to sustain FoxP3 expression and differentiation of naive $T$ cells into Tregs [6,7]. Shortly thereafter, observations were made indicating that TGF- $\beta$ functions in this regard could be deviated by IL- 6 to favor the differentiation of a new T-helper cell subset that produces IL-17 cytokine, hence the denomination Th17 cells [8,9]. ROR $\gamma \mathrm{t}$ was defined as the canonical transcription factor for the differentiation of Th17 cells [10]. This new subset received much attention owing to its involvement in inflammatory diseases [11]. In fact, IL-17 is known as a potent chemoattractant for neutrophils [12], and owing to this function the Th17 subset is now suspected to be involved in the initiation of multiple autoimmune disorders [11]. The question then is how IL- 6 meddles with TGF- $\beta$ to reform suppressive immunity into inflammatory responses. In other words, does IL-6 interfere with differentiation of naive $\mathrm{T}$ cells or convert terminally differentiated Tregs into Th17 cells? The process by which FoxP3 and ROR $\gamma t$ are able to accommodate this drastic transformation is not currently well understood. This is an important issue to address as the balance between Tregs and Th17 cells represents a feature of immense importance in the control of autoimmune diseases, tumor immunity and microbial infections.

\section{Summary of methods \& results}

In the current paper, Yang et al. illustrate the plasticity of what were believed to be terminally differentiated $\mathrm{CD}^{+}$Th17 and Tregs. They created an IL-17:red fluorescent protein (RFP) mouse, bred these mice to FoxP3:green fluorescent protein (GFP) mice and proceeded to polarize naive $\mathrm{CD}^{+} \mathrm{T}$ cells to become $\mathrm{Th} 17$ or induced Tregs (iTregs). Under Th17-polarzing conditions ( $\mathrm{aCD} 3, \mathrm{aCD} 28$, rIL-2, TGF- $\beta$ and IL-6), high levels of FoxP3 expression would not be expected. However, approximately $22 \%$ of the $\mathrm{CD}^{+} \mathrm{T}$ cells express FoxP3:GFP by day 2 of the stimulation. Moreover, $5 \%$ of the $\mathrm{CD}^{+} \mathrm{T}$ cells expressed both IL-17:RFP and FoxP3:GFP. Intriguingly, GFP levels decrease by day 4 of the stimulation, preliminarily indicating that a terminal differentiation decision may have been made by this point. This decrease
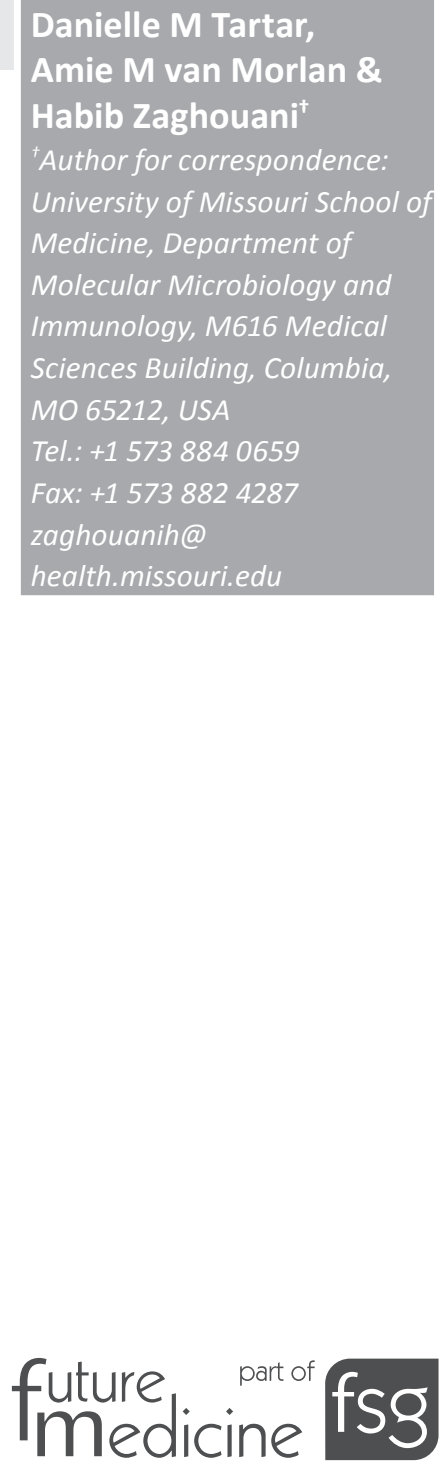
in FoxP3:GFP expression is not accompanied by an increase in apoptosis of the population, indicating that the cells were altering their phenotype rather than dying.

Naive $\mathrm{CD}^{+} \mathrm{T}$ cells were also stimulated under iTreg polarization conditions (aCD3, aCD28, rIL-2, TGF- $\beta$, aIL- 4 and aIFN- $\gamma$ ) and double-positive IL-17:RFP+ FoxP3:GFP+ cells were sorted along with single-positive IL-17:RFP-FoxP3: GFP' cells. RT-PCR analysis was used to further examine these cell populations. Accordingly, both the FoxP3 ${ }^{+} \mathrm{IL}-17^{+}$ double-positive population and the FoxP3 ${ }^{+}$ single-positive population expressed similar levels of FoxP3 transcript. Despite the presence of FoxP3, FoxP3 ${ }^{+} \mathrm{IL}-17^{+}$double-positive cells expressed higher levels of Th17-specific genes (IL-17, ROR $\gamma t$ and $R O R \alpha$ ) than their Fox $3^{+}$single-positive counterparts, indicating the induction of dual transcriptional programs in these cells. In the future, it would also be of interest to measure the expression of Tregspecific genes (TGF $\beta, I L-10$ and GITR, among others) in the FoxP3 ${ }^{+} \mathrm{IL}-17^{+}$double-positive relative - FoxP3 ${ }^{+}$single-positive cells. These in vitro findings collectively indicate that the pathways involved in a cell's decision to become a Treg or Th17 cell may not be as mutually exclusive as was previously believed.

The authors followed their in vitro findings by demonstrating that double-positive FoxP $3^{+} \mathrm{IL}-17^{+}$cells are generated in vivo just as they are in vitro, again indicating the possibility for the induction of dual transcriptional programs during differentiation of naive T cells into Tregs or Th17 cells.

To clarify the role of FoxP3 in the FoxP $3^{+} \mathrm{IL}-17^{+}$ double-positive cells, a FoxP3-expressing retrovirus was used. OT-II T cells were activated under Th17-polarizing conditions and were transduced with the FoxP3:IRES:GFP-containing retrovirus on day 1. Cells expressing the FoxP3:IRES:GFP construct showed a dramatic reduction in the Th17-specific genes $I L-17, I L-17 F$ and $I L-21$, but no noticeable effect on $R O R \gamma t$ or $R O R \alpha$ was observed. This indicates that the coexpression of FoxP3 somehow diminishes IL-17 expression without effecting expression of the canonical transcription factor ROR $\gamma \mathrm{t}$.

Finally, the authors demonstrate that 'differentiated' iTreg cells can be induced to lose FoxP3 expression and to produce IL-17 if they are restimulated with IL- 6 and TGF- $\beta$. They determined that TGF- $\beta$ and IL- 6 (either with or without IL-1 and IL-23) were able to drastically reduce the expression of FoxP3 while increasing
IL-17 expression. This is the first illustration of reprogramming terminally differentiated cells and illustrates the possible inherent plasticity of CD4 ${ }^{+}$T-cell subsets.

Importantly, this process also appears to occur with purified natural Tregs (nTregs). Indeed, sorted nTregs from B6 mice were restimulated with IL-6, which again led to a decrease in FoxP3 expression and a concurrent increase in IL-17. These cells were shown to be nonsuppressive in vitro. Finally, the authors determined that STAT3, ROR $\gamma$ t and ROR $\alpha$ were all required for Th17-specific gene upregulation but that, of these genes, only STAT3 was required for FoxP3 downregulation.

\section{Discussion \& signficance}

The current dogma in $\mathrm{CD}^{+}{ }^{+} \mathrm{T}$-cell differentiation views the development of T-helper subsets as a one-way road in which a naive T cells, upon activation, will evolve as a Th1, Th2, Treg or Th17 cell. The findings of Yang et al., however, show that a fraction of differentiating cells under Treg or Th17 conditions undergo a transitional double phenotypic (FoxP $3^{+} \mathrm{IL}-17^{+}$) state that ultimately resolves into a committed single Treg or Th17 phenotype. The discovery of FoxP $3^{+} \mathrm{IL}-17^{+}$cells suggests that the signals for differentiation may be more promiscuous than was previously thought and provides plasticity in molecular programming suitable for environmental control of subset commitment. More intriguingly, however, the paper shows that such plasticity extends to committed $\mathrm{T}$ cells. Indeed, the authors describe the reprogramming of terminally differentiated Tregs (both natural and induced) into Th17 cells, indicating that the road towards Treg differentiation is really more of a two-way road, a circumstance that can exacerbate autoimmunity or restore tumor responses.

\section{Future perspective}

The current paper by Yang et al. revealed a plasticity in the programming of the Treg/Th17 subset development that is operative during differentiation as well as after full commitment. This is illustrated by the presence of transitional FoxP $3^{+} \mathrm{IL}-17^{+}$cells during differentiation to either phenotype and the reprogramming of fully committed Tregs into Th17 cells. Whether this plasticity is operative in all T-helper cell pathways remains to be defined. In addition, further research needs to be carreid out to determine if reciprocal reprogramming of Th17 cells into Tregs can take place. 
These observations also raise a question as to whether expression of a particular phenotype represents a terminally differentiated cell or merely a transient state.

At the functional level, it would be of great significance to determine whether such plasticity can be shifted to reverse autoimmunity or eradicate tumors. Can approaches be defined to influence subset reprogramming in vivo? If so, this would add effectiveness to reversal of autoimmunity if one could reprogram pathogenic $\mathrm{T}$ cells into suppressor cells and clearance of microbes or eradication of tumors if suppressor cells could be converted into effector lymphocytes. In this regard, the paper moves the field both at the basic science level for better understanding of T-helper cell differentiation and at the clinical practice level for the design of effective approaches against autoimmunity, infection and tumor eradication.

\section{Financial \& competing interests disclosure}

This work was supported by grants RO1AI48541, RO1 DK65748, RO1 NS37406, RO1 NS057194 and R21AI68746 from NIH (to Habib Zaghouani) and by the JLavenia Edwards Chair endowment (to Habib Zaghouani). Danielle M Tartar was supported by a Life Sciences Fellowship from the University of Missouri. The authors have no other relevant affiliations or financial involvement with any organization or entity with a financial interest in or financial conflict with the subject matter or materials discussed in the manuscript apart from those disclosed.

No writing assistance was utilized in the production of this manuscript.

\section{Executive summary}

- Naive CD4+ T cells can differentiate into Th1, Th2, Treg or Th17 cells. Treg cells are immunosuppressive while Th1, Th2 and Th17 cells are effector cells.

- The paper under evaluation revealed a plasticity in subset programming and identified transitional cells expressing mixed Treg/Th17 phenotype during differentiation. The authors were also able to reprogram fully differentiated Treg cells and convert them into Th17 lymphocytes.

- The observations open avenues to determine whether such plasticity is common for all T-helper cell pathways and to evaluate the functional significance of subset reprogramming as it pertains to modulation of autoimmunity, clearance of microbial infections and eradication of tumors.

\section{Bibliography}

1 Yang XO, Nurieva R, Martinez GJ et al.: Molecular antagonism and plasticity of regulatory and inflammatory $\mathrm{T}$ cell programs. Immunity 29, 44-57 (2008).

2 Asano M, Toda M, Sakaguchi N, Sakaguchi S: Autoimmune disease as a consequence of developmental abnormality of a T cell subpopulation J. Exp. Med. 184, 387-396 (1996).

3 Sakaguchi S, Sakaguchi N, Asano M, Itoh M, Toda M: Immunologic self-tolerance maintained by activated $\mathrm{T}$ cells expressing IL-2 receptor $\alpha$-chains (CD25). J. Immunol. 155, 1151-1164 (1995).

4 Fontenot JD, Rasmussen JP, Williams LM, Dooley JL, Farr AG, Rudensky AY: Regulatory $\mathrm{T}$ cell lineage specification by the forkhead transcription factor FoxP3. Immunity 22, 329-341 (2005).
5 Shevach EM: From vanilla to 28 flavors: multiple varieties of $\mathrm{T}$ regulatory cells. Immunity 25, 195-201 (2006).

6 Wu Y, Borde M, Heissmeyer V et al.: FoxP3 controls regulatory $\mathrm{T}$ cell function through cooperation with NFAT. Cell 126, 375-387 (2006).

7 Fantini MC, Becker C, Montelone G, Pallone F, Galle PR, Neurath MF: Cutting edge: TGF- $\beta$ induces a regulatory phenotype in $\mathrm{CD}^{+}{ }^{+} \mathrm{CD} 25$ - $\mathrm{T}$ cells through FoxP3 induction and down-regulation of Smad7. J. Immunol. 172, 5149-5153 (2003).

8 Chen WJ, Jin W, Hardegen $\mathrm{N}$ et al.: Conversion of peripheral $\mathrm{CD} 4^{+} \mathrm{CD} 25^{-}$naive $\mathrm{T}$ cells to $\mathrm{CD} 4{ }^{+} \mathrm{CD} 25^{+}$regulatory $\mathrm{T}$ cells by TGF- $\beta$ induction of transcription factor FoxP3. J. Exp. Med. 198, 1875-1886 (2003).

9 Park H, Li Z, Yang XO et al.: A distinct lineage of CD4 $\mathrm{T}$ cells regulates tissue inflammation by producing interleukin 17 . Nat. Immunol. 6(11), 1133-1141 (2005).
10 Harrington LE, Hatton RD, Mangan PR et al.: Interleukin 17-producing CD $4^{+}$ effector $\mathrm{T}$ cells develop via a lineage distinct from the Thelper 1 and 2 lineages. Nat. Immunol. 6(11), 1123-1132 (2005).

11 Ivanov II, McKenzie BS, Zhou L et al.: The orphan nuclear receptor ROR $\gamma \mathrm{t}$ directs the differentiation program of proinflammatory $\mathrm{IL}-17^{+} \mathrm{T}$ helper cells. Cell 126, 1121-1133 (2006).

12 Ouyang W, Kolls JK, Zheng Y: The biological functions of $T$ helper 17 effector cytokines in inflammation. Immunity 28, 454-467 (2008).

13 Carlson T, Kroenke M, Rao P, Lane TE, Segal B: The Th17-ELR ${ }^{+}$CXC chemokine pathway is essential for the development of central nervous system autoimmune disease. J. Exp. Med. 205, 811-823 (2008). 